\title{
Eusscorppins Bicentennial
}

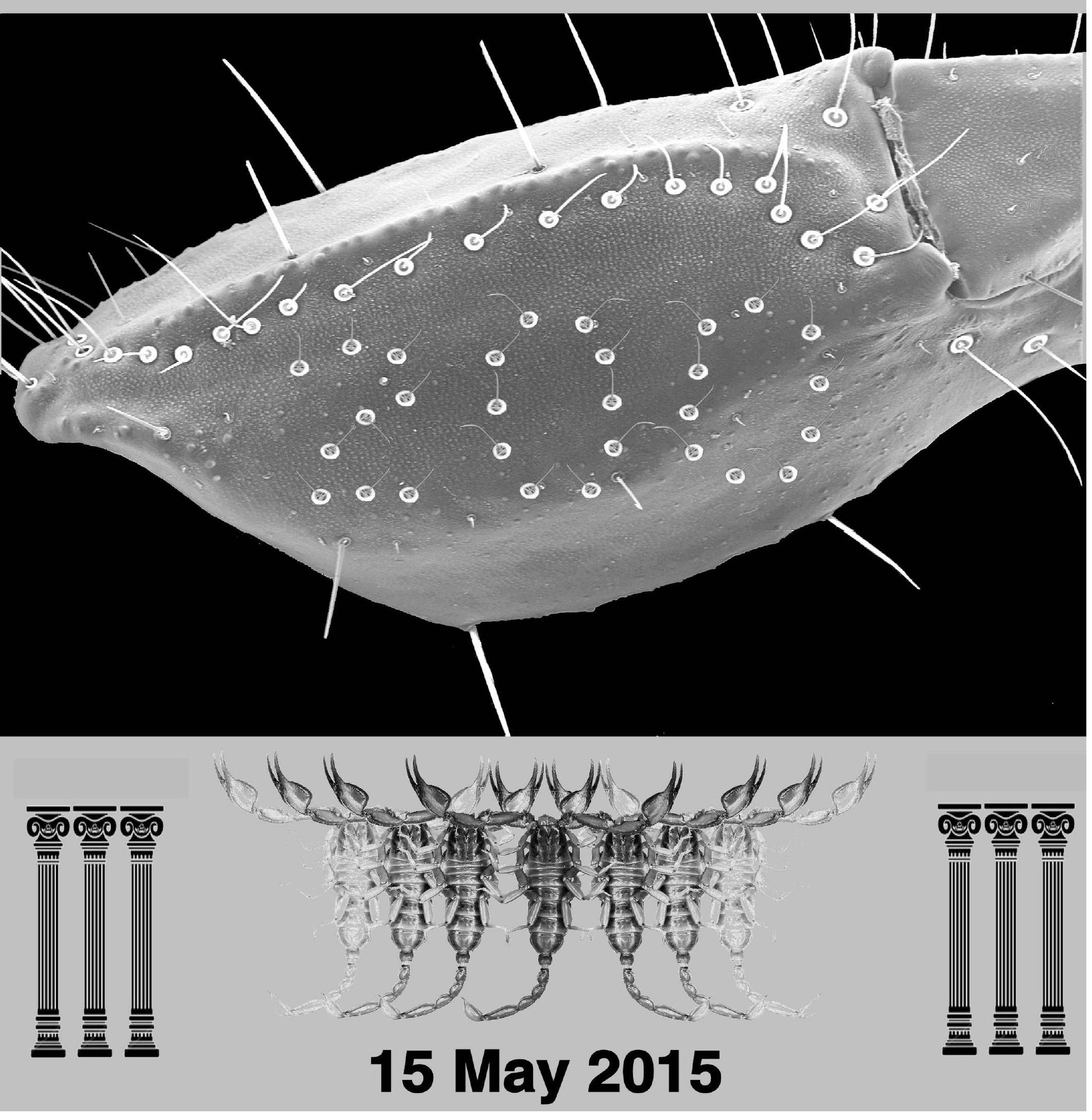




\section{AUTHORS}

1. Acosta. L.E.: 2

2. Ahmed, Z.: 53, 88, 108, 168

3. Armas, L.F. de: 14, 18, 22, 33, 66, 116, 127, 132, 172

4. Ayrey, R.: 86, 111, 114, 130, 148, 166, 176, 183, 188, 194

5. Balanika, K.: 182

6. Baldazo-Monsivaiz, J.G.: 197

7. Baptista, C.: 35

8. Benli, M.: 61, 164

9. Bost, K.C.: 12,17

10. Bouderka, A.: 182

11. Boumezzough, A.: 113

12. Brewer, M.S.: 41, 45, 49

13. Bryson, R.W. Jr.: 140

14. Cao, Z.: 118,153

15. Chazal, L.: 92, 106

16. Cloudsley-Thompson, J. L.: 124

17. Çolak, M: 30, 171

18. Colombo, M.: 36, 87, 117

19. Cozijn, M. A. C.: 133, 178, 179

20. Crucitti, P.: 9

21. David, D.: 151

22. Di, Z.: 104, 118, 153

23. Dinamithra, N.P.: 155

24. Duhem, B.: 110

25. Dupré, G.: 50

26. Edwards, D.: 8.

27. El-Hennawy, H. K.: 119

28. Ezatkhah, M.: 131

29. Van der Ende, T.: 77

30. Facheris, D.: 52

31. Farley, R.: 120

32. Fet, V.: 1, 3, 5, 11, 13, 17, 20, 23, 24, 29, 38, 39, 40, 41, 45, $48,49,65,67,69,71,74,78,79,82,95,99,101,109,112$, $121,131,134,139,143,147,159,161,165,169,170,185$, 190, 192, 193, 199, 200

33. Fomichev, A.A.: 136

34. Gaffin, D.D.: 12, 17, 122

35. Gantenbein, B.: 4, 17

36. García H., L.F.: 60, 63, 64

37. Geniez, P.: 19

38. Gharkheloo, M.M.: 147

39. Graham, M.R.: 42, 46, 48, 51, 57, 185

40. Gromov, A.V.: 4, 17

41. He, Y.-W.: 118 
42. Henderickx, H. 137

43. Hypolite, F. 102

44. Javed, S. M. M.: 98, 107

45. Jiao, G.-B.: 80,84

46. Johnston, C.: 17

47. Kaltsas, D.: 44, 55, 56,

48. Karataş, A.: 7, 25, 43, 171

49. Karataş, Ah.: 7

50. Kayedi, M. H.: 99

51. Kloock, C.: 21

52. Koç, H.: 76, 85

53. Kořínková, T.: 77, 189

54. Košulič, O.: 160

55. Kotsakiozi, P.: 161, 169, 190

56. Kovařík, F.: 9, 10, 15, 16, 20, 26, 27, 28, 34, 38, 39, 53, 54, 58, $65,67,69,74,77,78,79,82,88,95,97,99,108,109,115$, $126,127,128,129,131,132,138,139,141,142,143,149$, $150,155,157,158,159,160,163,168,170,173,175,177$, $178,186,187,189,191,192,193,195,196,198,200$

57. Král, J.: 77, 189

58. Kularatne, S.A.M.: 155

59. Kunt, K. B.: 85

60. Kürtüllü, M.: 43

61. Kutcher, S.R: 8

62. Leguin, E.-A.: 75, 89, 110, 123, 135, 151

63. Li, W.-X.: 118, 153

64. Liu, H.: 153

65. Lourenço, W.R.: 4, 19, 32, 59, 73, 75, 83, 89, 102, 110, 113, $123,124,135,137,146,151,156$

66. Lowe, G.: 4, 8, 23, 90, 91, 93, 96, 100, 103, 112, 138, 173, 191,196

67. Madden, H.: 145

68. Martín-Frías, E.: 22

69. Mazuch, T: 126

70. McReynolds, C.N.: 17, 144

71. Mirshamsi, O.: 154

72. Mirza, Z.A.: 98,107

73. Mosseichik, Yu.V.: 13

74. Mylonas, M.: 44, 55.

75. Nagasena, I. I.: 155

76. Navidpour, S.: 65, 67, 69, 74, 78, 99, 109, 131, 139, 170, 192

77. Nayebzadeh, H. H.: 99

78. Neff, D.P.A.: 41,49

79. Norton, M.L.: 41,49

80. Ochoa, J.A.: 2

81. Paniagua-Solís, J.F.: 22. 
82. Parmakelis, A.: 161, 165, 169, 182, 190

83. Pirali-Kheirabadi, K: 78

84. Plíškova, J.: 158, 173, 196

85. Porterfield, C.: 17

86. Porterfield, J.Z.: 17

87. Qi, J.-X.: 32

88. Ranawana, K.B.: 155

89. Rao, K. T.: 98

90. Rein, J.O.: 6, 94

91. Reyes Lerma, A.C.: 189

92. Rivera, M.J.: 180

93. Roncallo, C.A.: 62, 68, 72, 105

94. Rossi, A.:167

95. Rojas-Runjaic, F.J.M.: 47

96. Sanap, R.V.: 98, 107

97. Sánchez, A.J.: 180

98. Santiago-Blay, J.A.: 35

99. Seiter, M.: 178, 196

100. Shcherbakov, D.E.: 13, 121

101. Sivansuthan, S.: 155

102. Soleglad, M.E.: 1, 3, 5, 11, 13, 17, 20, 23, 24, 29, 40, 41, 45, $49,57,65,67,69,71,74,78,79,82,95,99,101,112,114$, $121,131,134,139,143,159,160,161,165,170,183,185$, $193,194,200$

103. Št’áhlavský, F.: 77, 158, 173, 196

104. Stathi, I.: 44, 101, 161, 165, 169, 190

105. Steinmetz, S.B.: 12

106. Stewart, A. K.: 37

107. Stockmann, M.: 124

108. Sziszkosz, N.: 182

109. Tampal, F.: 98

110. Teruel, R.: $14,60,62,63,64,68,70,72,92,94,97,105,106$, $115,125,127,132,133,145,177,178,179,180,186,187$, $192,197,198$

111. Tietz, A.K.: 70

112. Touloun, O.: 113

113. Tropea, G.: 161, 162, 167, 169, 174, 182, 184, 190, 199

114. Trujillo, R.E.: 172

115. Turiel, C.: 177,181

116. Upadhye, R.: 107

117. Vignoli, V.: 9

118. Walvoord, M.E.: 17

119. Webber, M. M.: 140

120. Wu, Y.-L.: 118,159

121. Yağmur, E.A.: 76, 85, 95, 109, 143, 159, 161, 164, 167, 191, 200 
122. Yalçın, M.: 76

123. Yamashita, T.: 17

124. Yeşilyurt, F.: 184

125. Yigit, N.: 61

126. Ythier, E.: 89,124

127. Zambre, A.: 107

128. Zhang, L.: 81

129. Zhu, L.: 153

130. Zhu, M.-S.: 32, 80, 81, 84, 104

131. Zonstein, S. L.: 134

Cover photo: The cover image is based on Anuroctonus phaiodactylus (Garrison, Utah) SEM taken by Kelly Anne Daniel. 


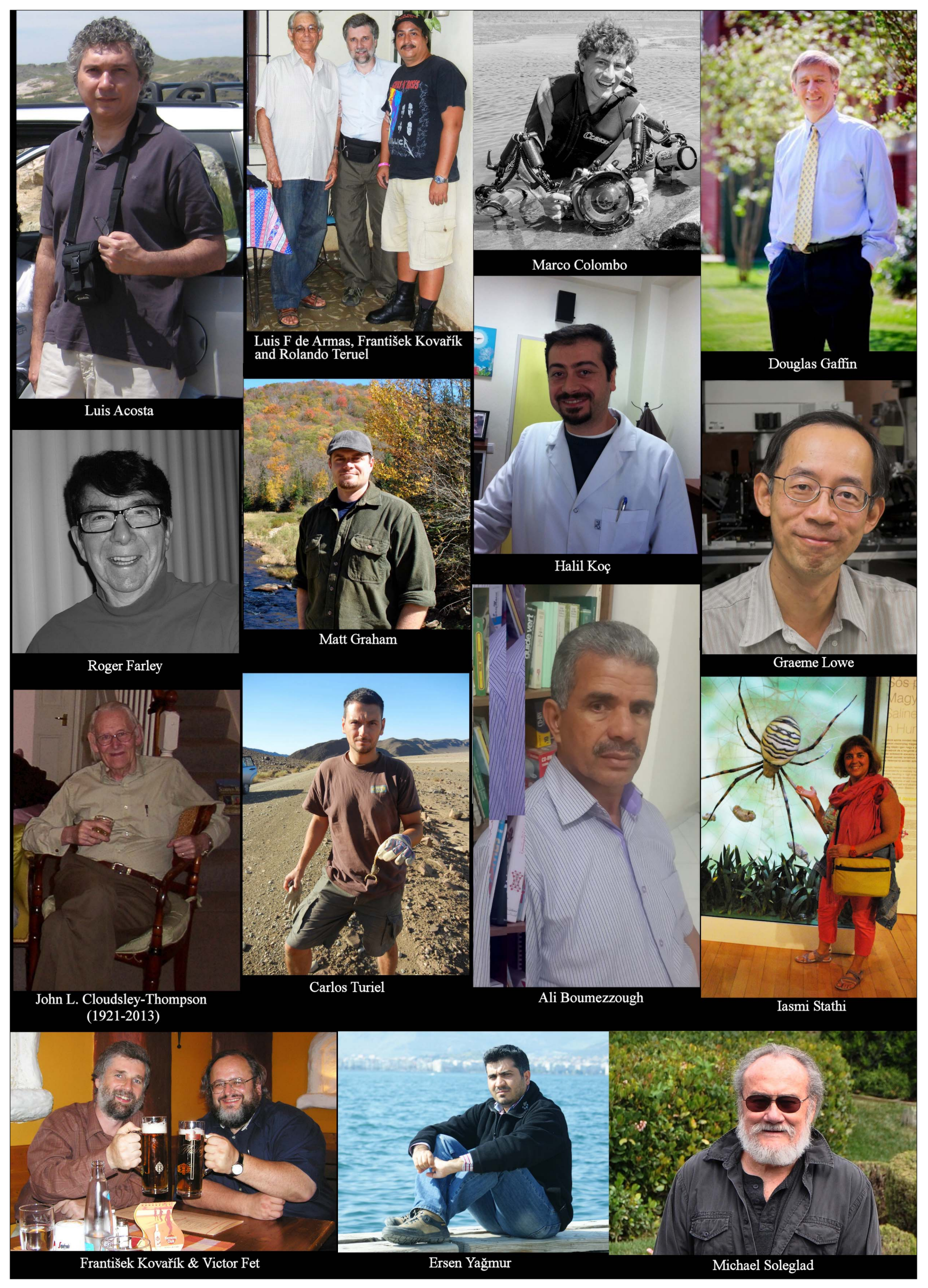




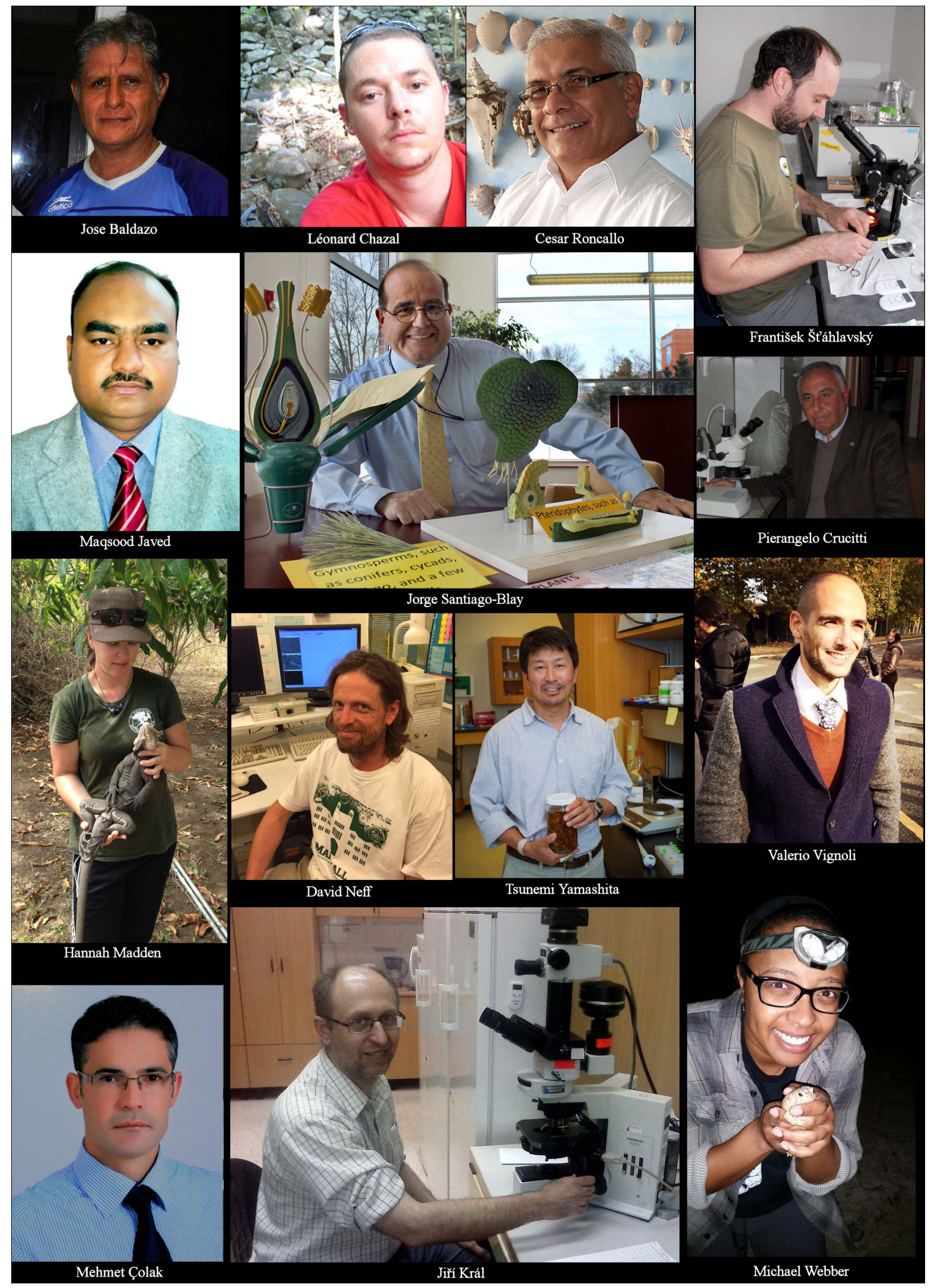




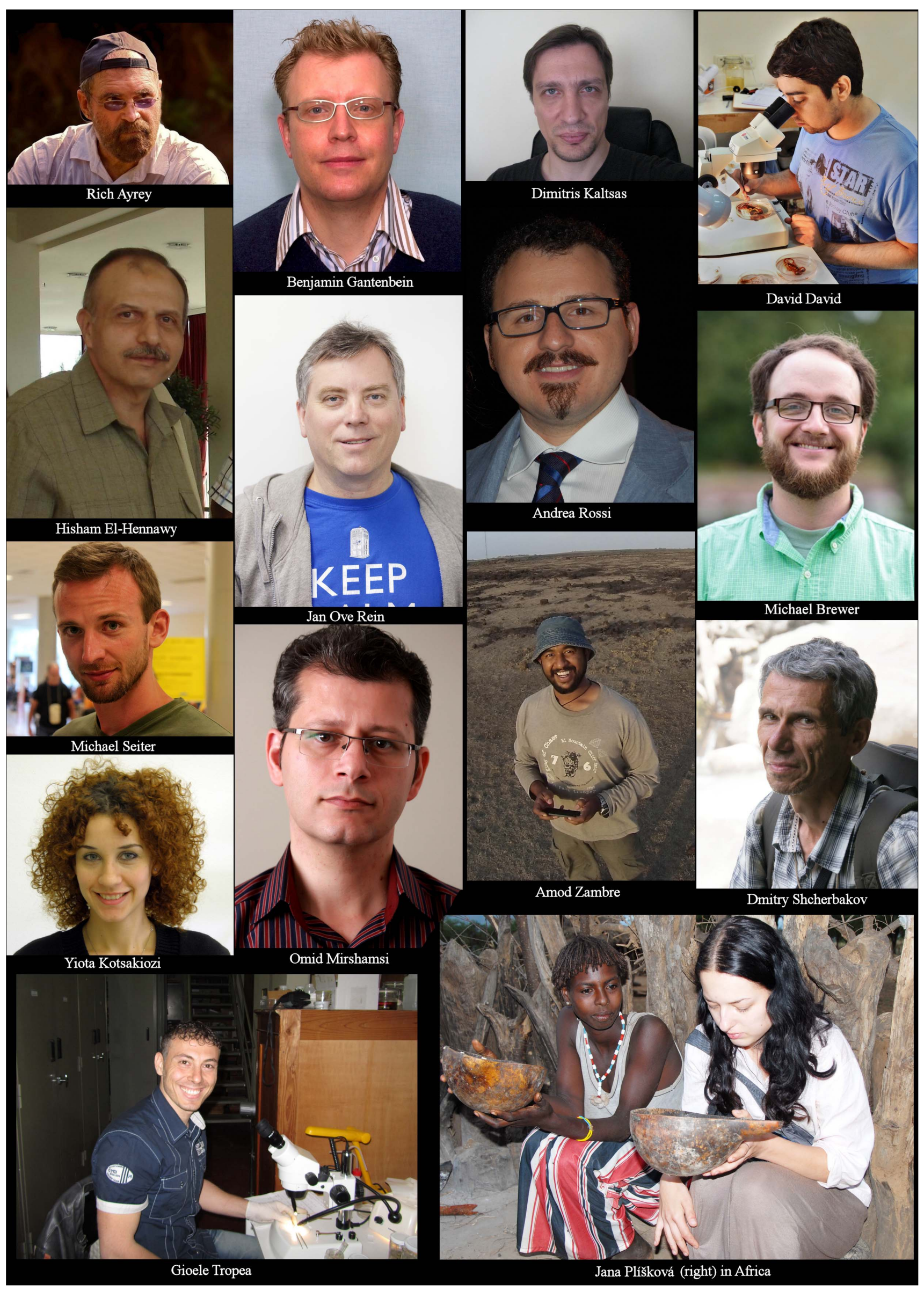




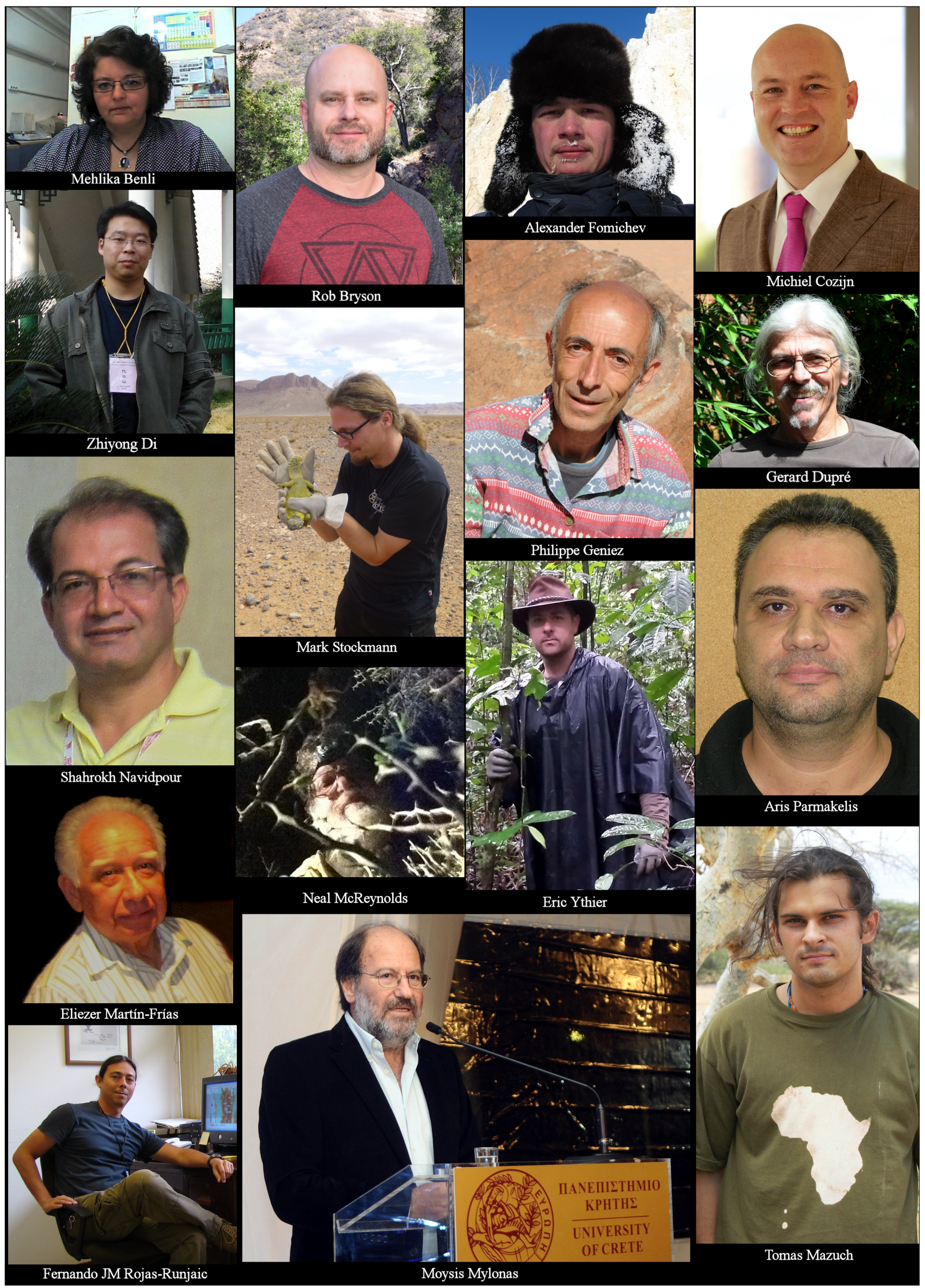




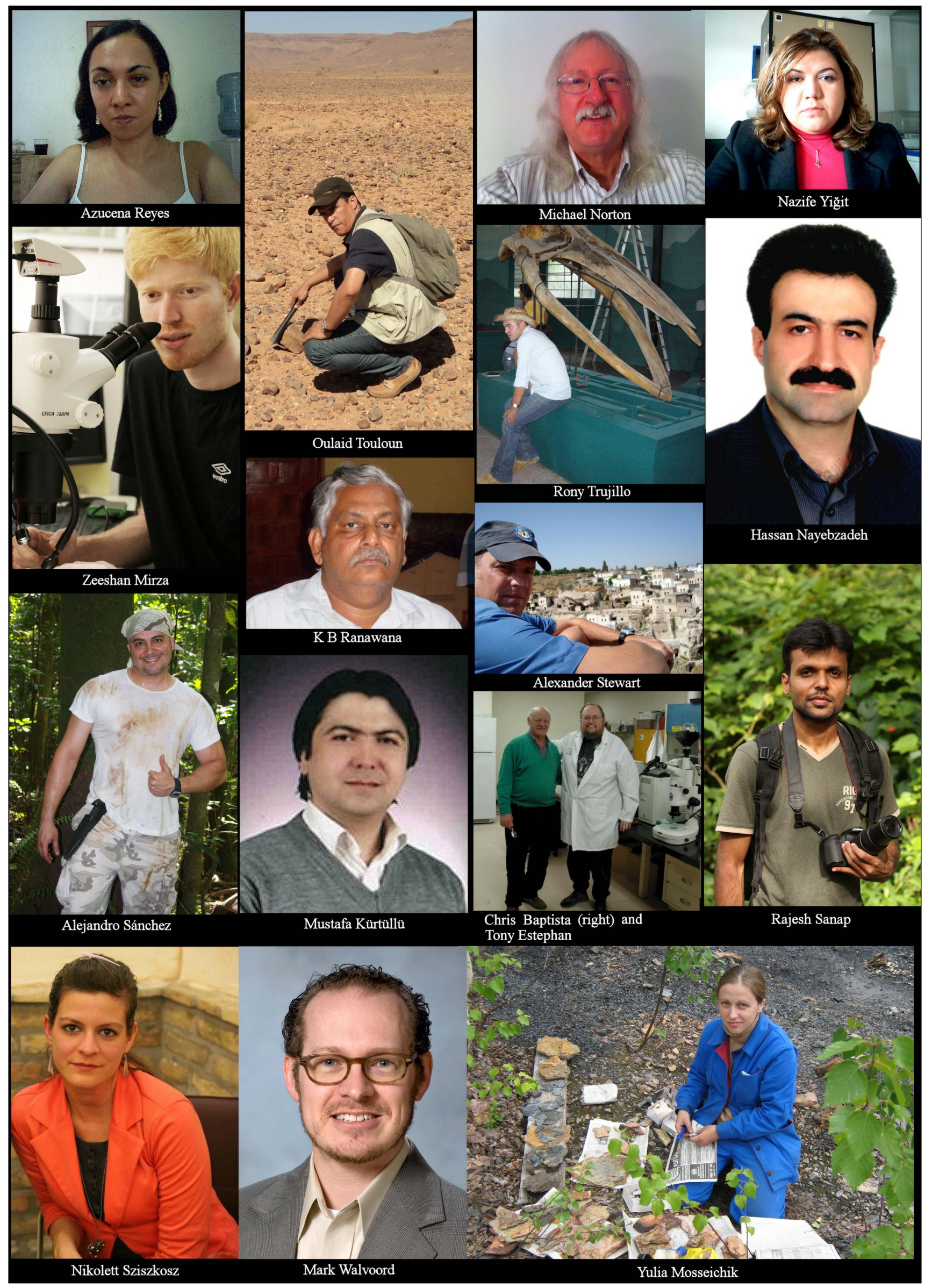




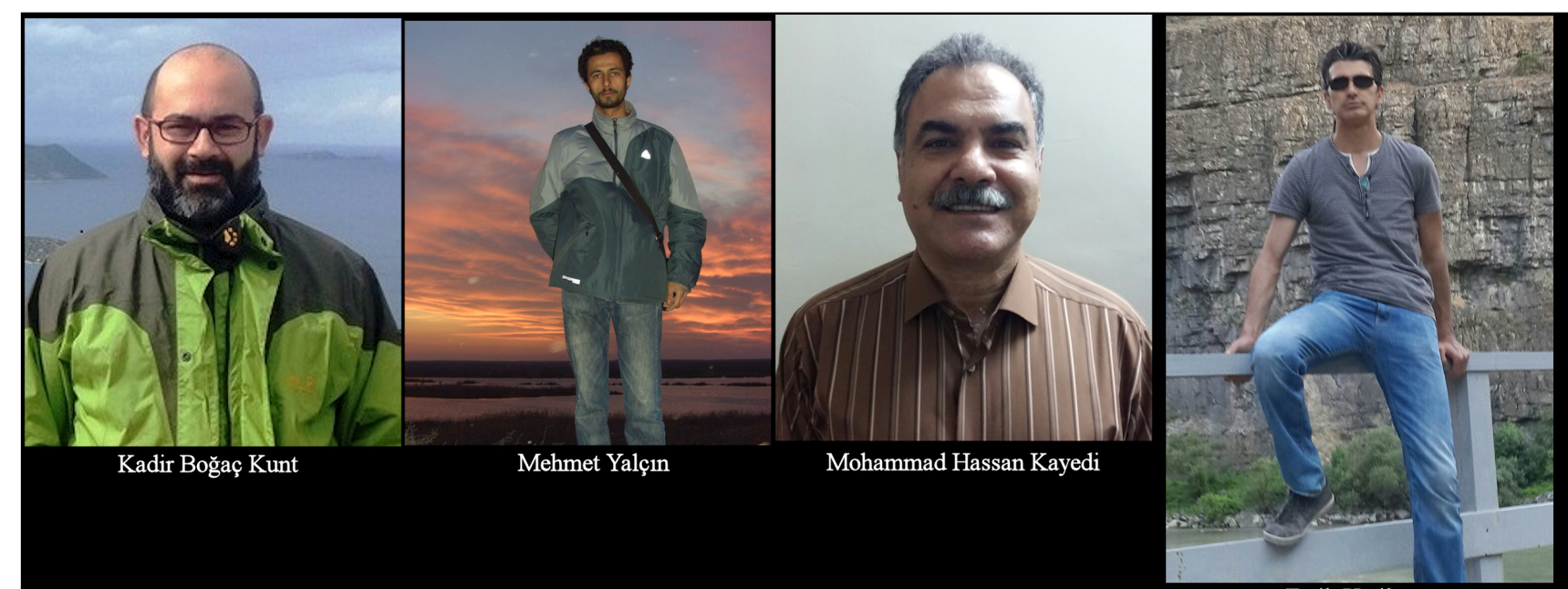

Fatih Yeşilyurt
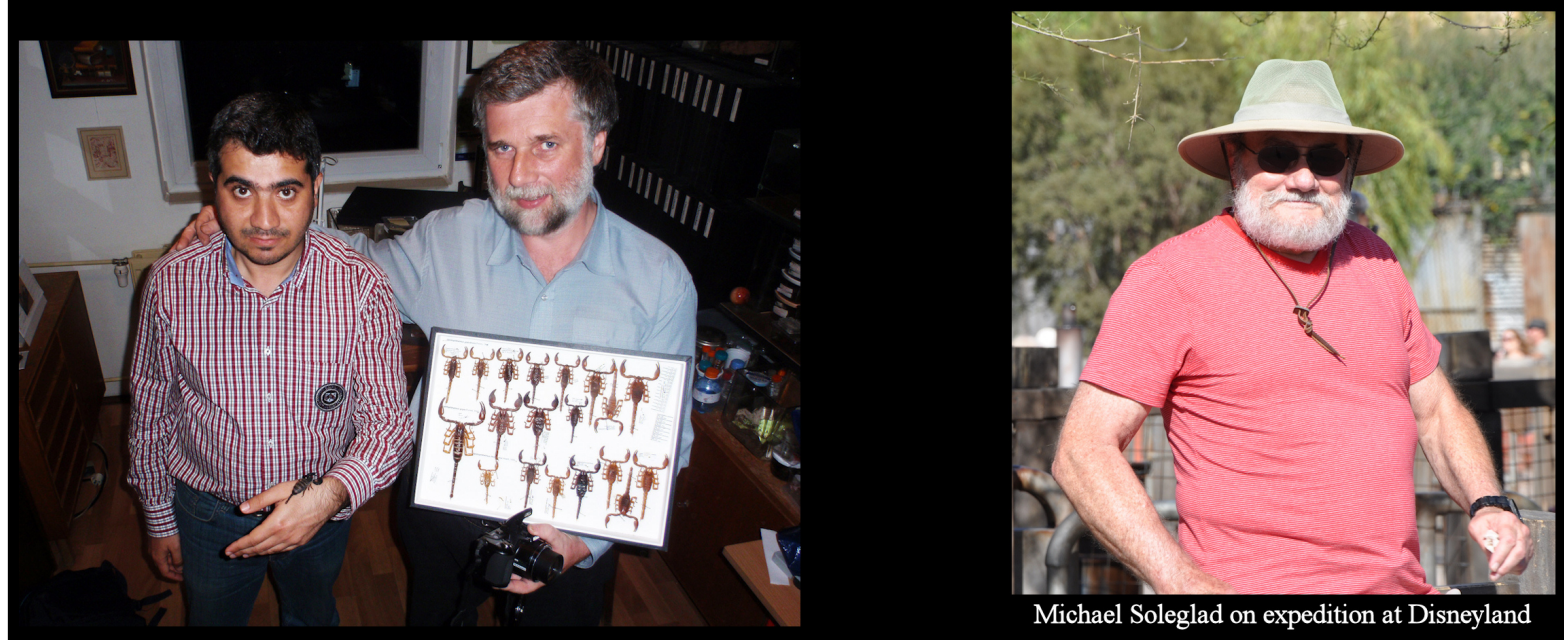

Ersen Yağmur and František Kovařik

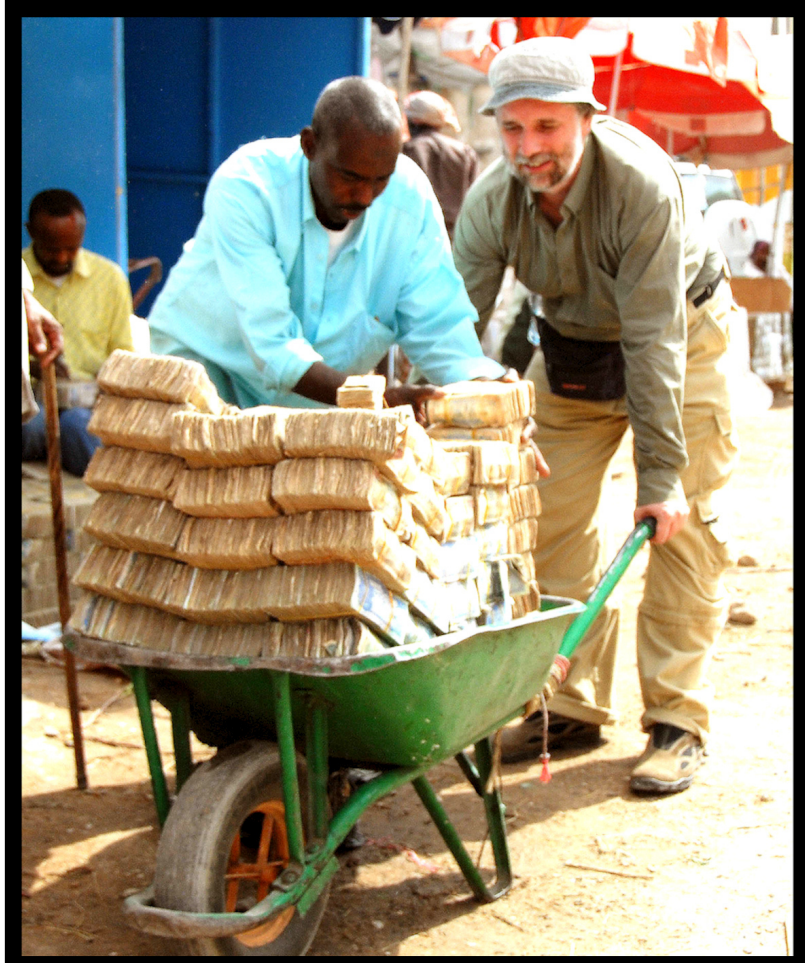

František Kovařik (right) with African money

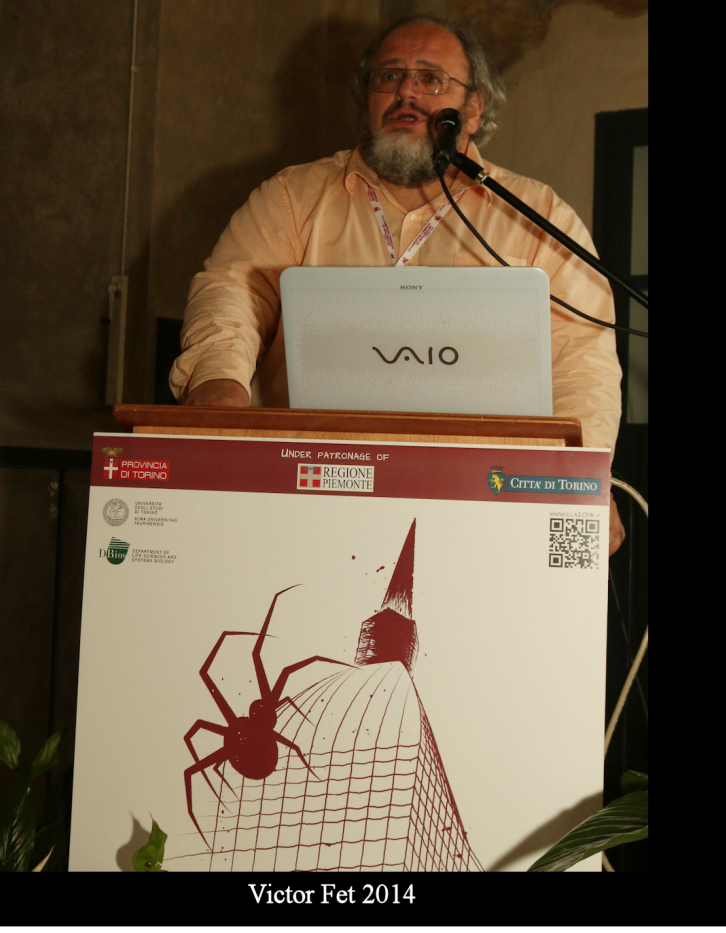




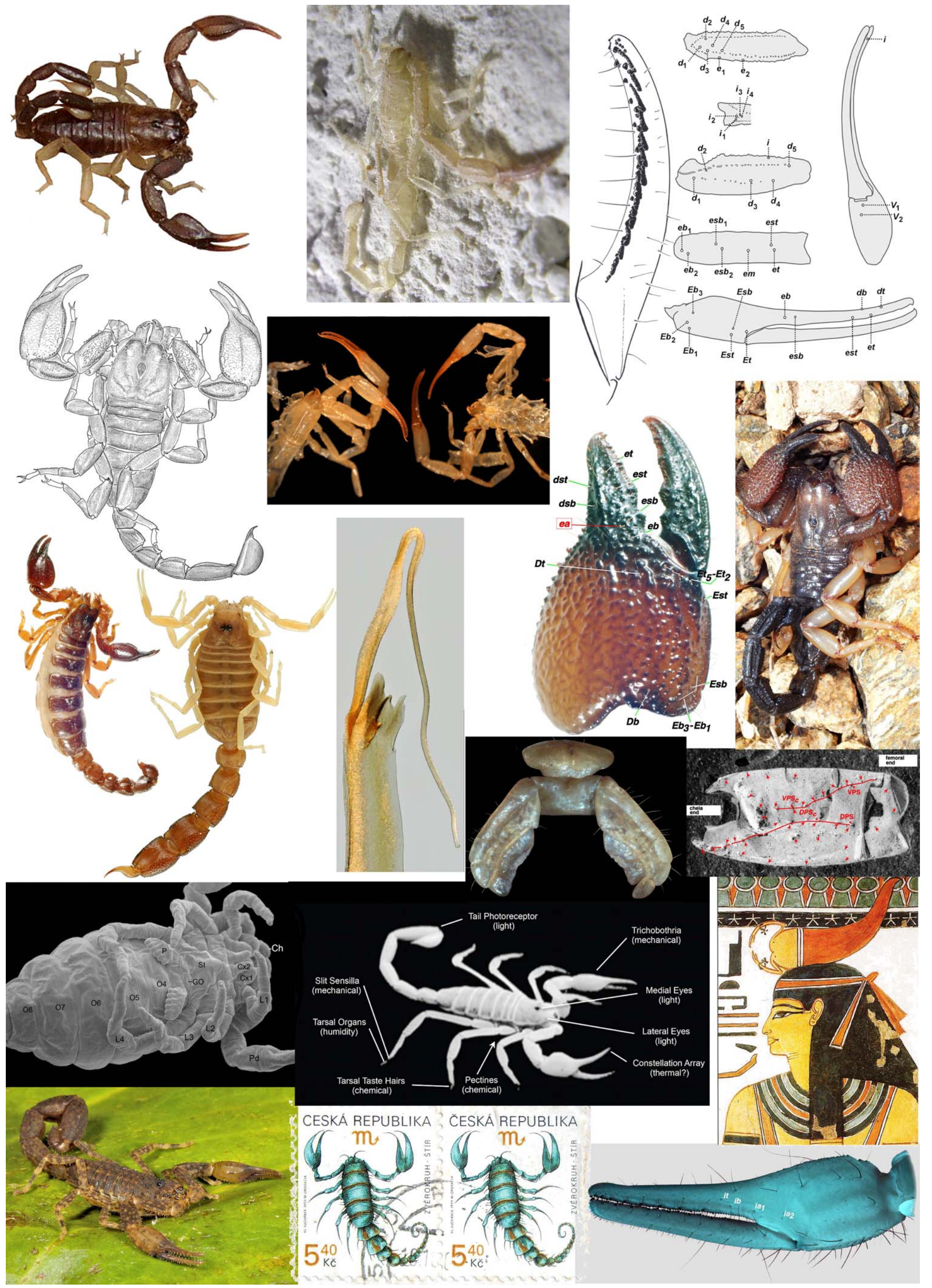




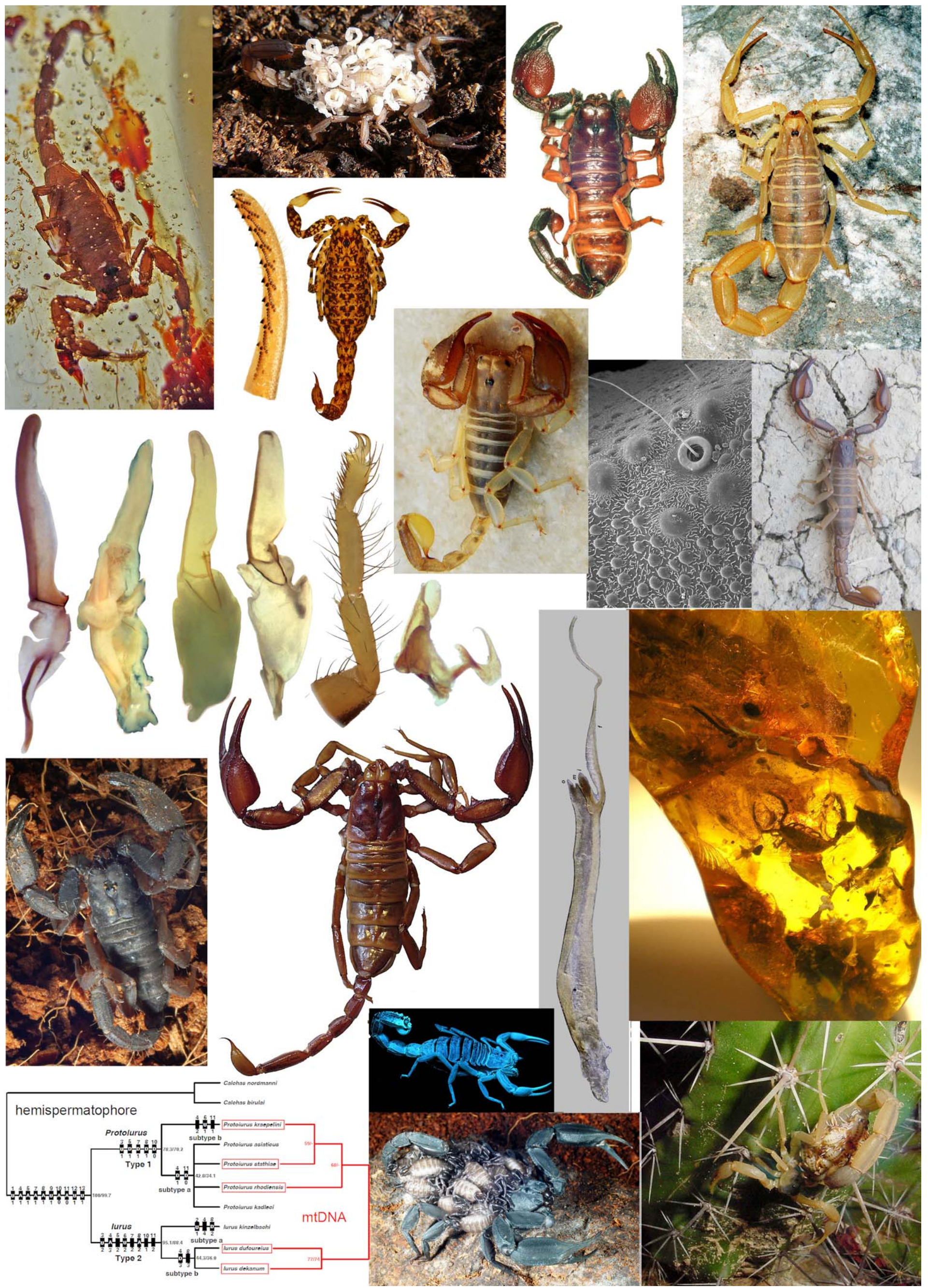




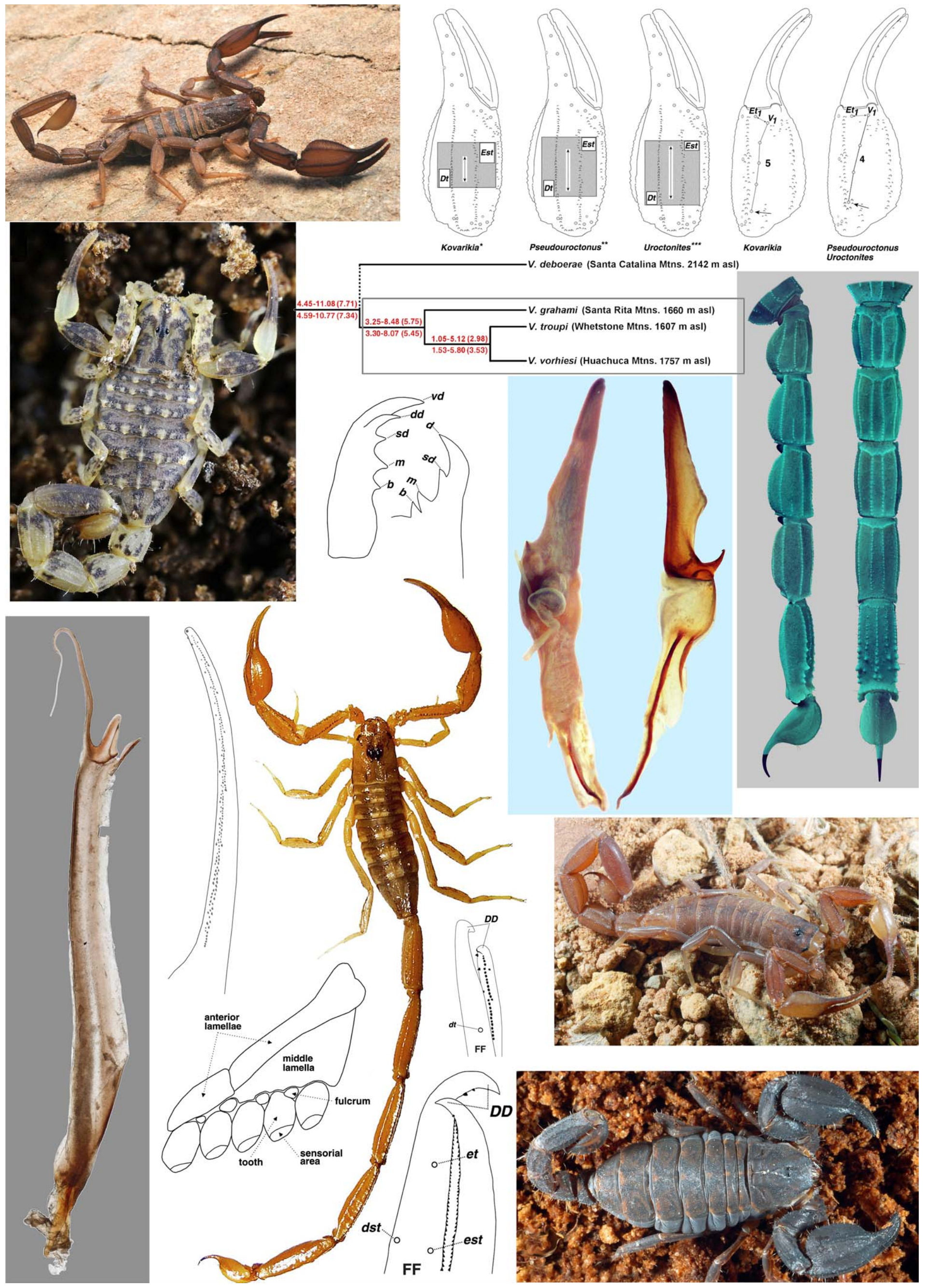




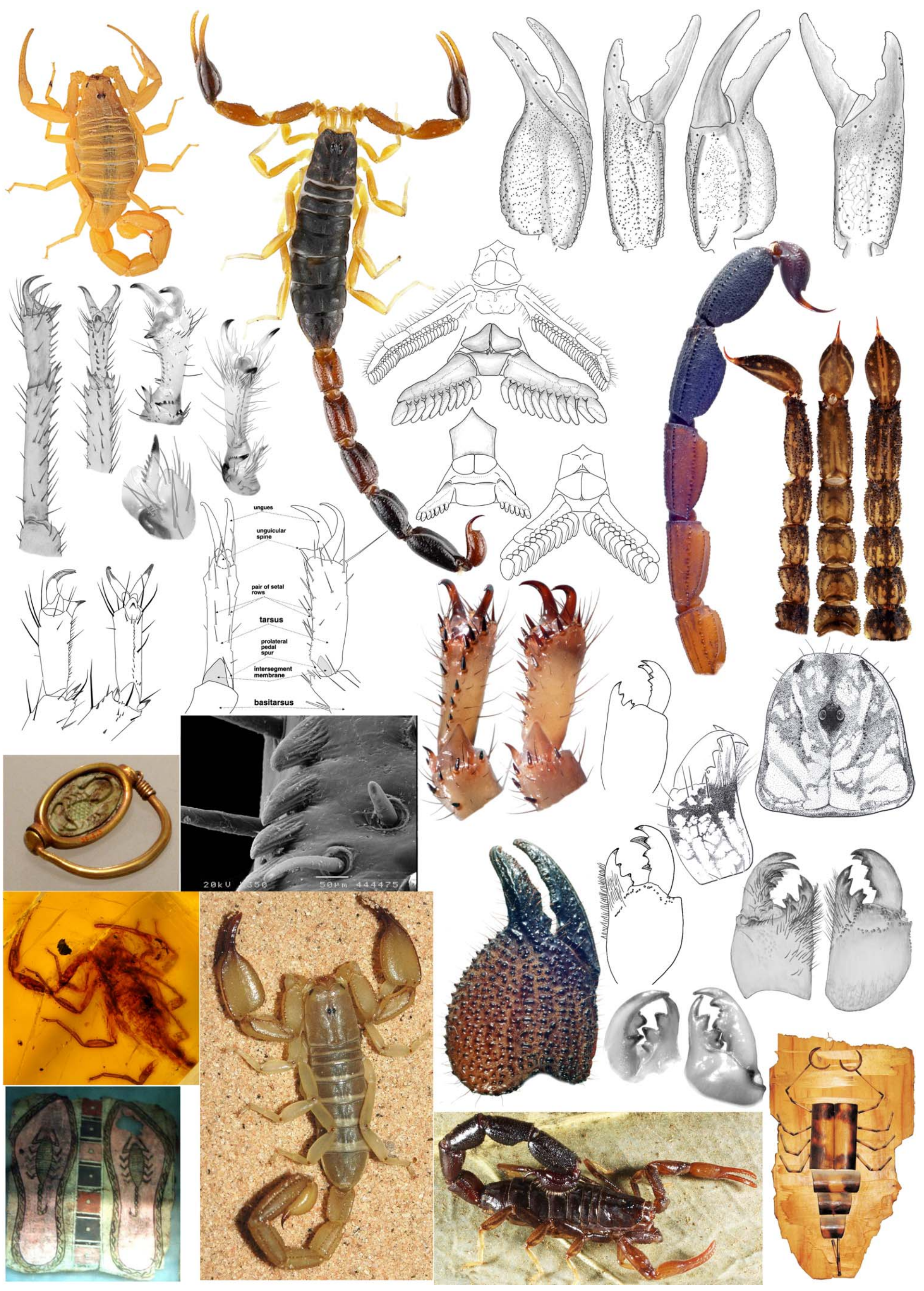

